\title{
Anesthesia for Cesarean Section: Retrospective Comparative Study
}

This article was published in the following Dove Press journal:

International Journal of Women's Health

\author{
Naser Al-Husban (ID) \\ Mohammad Sami Elmuhtaseb' \\ Hedaieh Al-Husban ${ }^{2}$ \\ Mohammed Nabhan (iD ${ }^{3}$ \\ Hamza Abuhalaweh ${ }^{3}$ \\ Yasmine Mohamed Alkhatib ${ }^{3}$ \\ Maysa Yousef ${ }^{3}$ \\ Bayan Aloran ${ }^{3}$ \\ Yousef Elyyan ${ }^{3}$ \\ Asma Alghazo $\mathbb{D}^{3}$ \\ 'Faculty of Medicine, The University of \\ Jordan, Amman, Jordan; ${ }^{2} \mathrm{Al}-\mathrm{Noor}$ \\ Fertility Center, Eye Specialty Hospital, \\ Amman, Jordan; ${ }^{3}$ Obstetrics and \\ Gynecology Department, Jordan \\ University Hospital, Amman, Jordan
}

Background: Cesarean section is a widely performed surgery.

Objective: To compare anesthetic types regarding feto-maternal outcomes.

Materials and Methods: Retrospective comparative study of 3599 cesarean sections (emergency and elective categories).

Results: Mean APGAR score was statistically higher in the spinal than general anesthesia among the emergency category, $\mathrm{P}=0.000$ and 0.026 , respectively, with no significant difference in the elective category. Estimated blood loss among the elective category was statistically significantly higher in the spinal than general anesthesia, $\mathrm{P}=0.001$. However, among the emergency category, it was significantly higher in the general than in spinal or epidural anesthesia, $\mathrm{P}=0.000$. Diclofenac sodium was used more after spinal than general anesthesia $(\mathrm{P}=0.000)$, with no significant difference between epidural and general or between epidural and spinal anesthesia. Pethidine hydrochloride (HCL) was used more after general than after spinal anesthesia $(\mathrm{P}=0.000)$. However, pethidine HCL use was not statistically significantly different between spinal and epidural anesthesia. In the elective category, paracetamol was requested more after spinal than epidural or general anesthesia, $\mathrm{P}=0.000$. No significant difference was seen between epidural and general anesthesia, $\mathrm{P}=$ 1.000. No statistically significant difference was found among the anesthetic types in both categories regarding tramadol HCL. Length of hospital stay, operative time and neonatal intensive care unit admission were not statistically different between anesthetic modes. In the emergency category, significantly higher percentage of patients were satisfied with and would recommend epidural anesthesia.

Conclusion: There was no statistically significant difference among the three types of anesthesia regarding neonatal intensive care admission and length of hospital stay for emergency and elective categories. APGAR score was higher with spinal than with general anesthesia in the emergency category with no significant difference in the elective category. More diclofenac sodium and paracetamol and less opioids were used after regional than after general anesthesia. Satisfaction was higher with epidural anesthesia.

Limitations: Retrospective and single centered.

Keywords: cesarean section, spinal, epidural, general, anesthesia, feto-maternal, outcome

\section{Introduction}

Physiological changes in pregnancy, including hematological, cardiovascular and respiratory changes, all increase the risks during cesarean sections (CS) and anesthetic management of a parturient is a challenge because it involves simultaneous care of both mother and baby. ${ }^{1-4}$

These risks and complications are related to the level of urgency. ${ }^{5}$ 
Clinical experience of the majority of anesthetists with general anesthesia (GA) in obstetrics is very low. GA is mostly conducted for emergency cesarean section due to the time factor dictated by fetal condition which usually precludes regional anesthesia. ${ }^{6}$

Technical placement of a spinal anesthetic (SA) is easier than an epidural block in epidural anesthesia (EA). Onset of action of spinal anesthesia is fast and it provides a reliable surgical anesthesia from the mid-thoracic level to the sacrum with a failure rate of less than $1 \%$. Moreover, SA was found to provide better and more costeffective anesthesia for uncomplicated, elective cesarean sections than EA. ${ }^{7,8}$

Cesarean section is a widely performed surgery with a rate of maternal mortality that is much higher than vaginal delivery and the leading causes of death are complications of preeclampsia, pulmonary thromboembolism, amniotic fluid embolism, obstetric hemorrhage and cardiac disease. ${ }^{9}$

\section{Aim of the Study}

This study aimed to investigate the influence of the type of anesthesia on the different feto-maternal outcomes. Since the choice of anesthesia for cesarean delivery is very important for both maternal and fetal outcomes and is also related to the post-operative course, it needs to be studied in depth.

\section{Materials and Methods}

A retrospective comparative study was conducted between the three types of anesthesia; spinal, epidural and general. We reviewed all cesarean deliveries of women between January 2016 and December 2018. We excluded multiple pregnancies, known medical diseases, those who had cesarean hysterectomy and those who had any missing clinical data. It was conducted at a university teaching hospital. There were two main categories of cesarean sections, elective and emergency. The three types of anesthesia within each category were compared in relation to the duration of cesarean section, estimated blood loss (EBL), APGAR score at 1 and 5 minutes, duration of postoperative hospital stay (LOS), neonatal intensive care unit admission (NICU), analgesic requirements (diclofenac sodium, pethidine hydrochloride (HCL), paracetamol, morphine, tramadol HCL) and patient overall satisfaction and recommendation.

The indications for the cesarean section were divided into two main categories, elective and emergency. The elective indication was defined as any cesarean that was done electively with a previously scheduled admission and surgery. This definition included fetal and maternal indications as well as maternal request. The emergency indications included all cesarean sections that were performed for fetal and maternal indications, failed vacuum or forceps delivery and those who were already scheduled for an elective cesarean but presented before their cesarean date due to feto-maternal indications, e.g., a women with three previous cesarean sections who presented contracting before her scheduled admission and cesarean section.

Patients had a similar standardized general, spinal or epidural anesthetic according to the hospital protocols. All patients were evaluated before surgery or antenatally. They were all fasting in the elective category, and no premedications were given. Fasting status was not identified in the emergency category. Anesthesia type was approved after counselling and with written informed consents. GA patients had brief standard preoperative monitoring then induction with fentanyl and propofol. Furthermore, the muscle relaxant cisatracurium was used with endotracheal tube as indicated and isoflurane maintenance with controlled mechanical ventilation. Additionally, neostigmine and atropine were used as reversal agents in patients who received muscle relaxants. At the end of surgery, patients were easily extubated. SA was performed at the level of L3-L4, L4-L5 lumbar spines. Patients were given bupivacaine $10.0 \mathrm{mg}$ with fentanyl $25 \mu \mathrm{g}$ intrathecally under aseptic technique. A test dose of $3 \mathrm{~mL}$ of $1 \%$ lidocaine $(30 \mathrm{mg})$ was administrated in epidural anesthesia then an infusion of $1 \mathrm{mg} / \mathrm{mL}$ bupivacaine and $2 \mu \mathrm{g} / \mathrm{mL}$ fentanyl. Epidural catheters were removed at the conclusion of surgery. Patients were usually maintained on intravenous fluids $150 \mathrm{~mL} / \mathrm{h}$, and any fluid deficit was replaced. The vast majority of the cases were anesthetized by senior anesthesia residents. The option to have general or spinal anesthesia was offered routinely (unbiased on the side of anesthetists) to all patients unless dictated by a medical condition. Epidural anesthesia was also offered to all laboring women or those undergoing induction of labor. It was only in very rare emergency occasions that a general anesthetic was recommended.

Cesarean section patients, whether elective or emergency, received prophylactic antibiotics after clamping the umbilical cord in the form of $2 \mathrm{~g}$ of cefazolin intravenously.

The duration of the cesarean section was defined as the time, in minutes, spent in the cesarean section from the 
beginning of the skin incision to the complete wound cover. This was recorded routinely for each cesarean section by the circulating theatre nurse.

The estimated blood loss $(\mathrm{mL})$ was routinely recorded in our hospital for all operations in a standardized way. This took into account the number of surgical pads used, the blood in the suction container, and any free blood or clots in the surgical field, theatre floor or on the surgical towels. The final EBL was routinely agreed upon by the primary surgeon, the assistant surgeon(s), the anesthetist (s) and the theatre nursing staff. The vast majority of cesarean sections were performed by 4 th and 5 th year residents except for complicated cases which necessitated supervision by a consultant. All patients had received an oxytocin bolus dose after the delivery of the baby as per the hospital policy.

The APGAR score was routinely recorded by the attending neonatal team who attended all cesarean sections as per the hospital protocol.

The duration of post-operative hospitalization, in days, was calculated as the number of days, excluding the day of surgery, spent in the hospital due to maternal surgical indication and not due to maternal medical, social and financial conditions or neonatal indication. This was because some women would wait in the hospital for their newborns if they were admitted for observation or any other indication.

The post-operative analgesic requirements were defined as the number of standardized parenteral dosages that were requested by the patient after discharge from the theatre till the discharge decision was made by her surgeon. The number of doses of the particular analgesia was primarily determined by the patient's request as there was no well-defined hospital analgesia protocol. Other factors play a part such as what type of injection was given and when it was given. This type of post-operative analgesia was applied and used in all patients allowing a fair comparison between the anesthetic types. Diclofenac sodium was given in intra-muscular (IM) injections of $75 \mathrm{mg}$, pethidine HCL was given in IM injections of $100 \mathrm{mg}$, paracetamol was given in intravenous (IV) infusion of $1000 \mathrm{mg}$, morphine sulfate was given as IM injections of $10 \mathrm{mg}$ and tramadol HCL was given as IM injection of $50 \mathrm{mg}$. However, oral analgesia was not studied as it was rarely prescribed by physicians because the patients rarely, if ever requested that type of analgesia, while still in the hospital. However, upon discharge, they were usually given both paracetamol and ibuprofen orally.
The overall women's satisfaction with the anesthetic mode was assessed subjectively by yes or no answers and whether or not they would recommend the same mode to a friend. It was done after discharge and when the patients were relatively well enough to reflect on their overall cesarean experience.

These data were collected using medical files (both paper and electronic), admission notes, clinic notes, operative notes, neonatal notes, and, if needed, telephone calls with patients.

Data were analyzed using SPSS 24 software. Frequency and percentage were calculated for the categorical data and analyzed using ANOVA test with post hoc Bonferroni and chi squared analysis as appropriate as we were comparing three types of anesthesia together. The level of significance was considered to be $95 \%$ $(\mathrm{p} \leq 0.05)$. Values stated are mean \pm standard deviation (SD) unless otherwise indicated.

The study obtained the institutional review board (IRB) approval at Jordan University Hospital, decision number 95/2019, dated 5/3/2019. The IRB committee considered that a written informed consent was not necessary due to the retrospective nature of the study. However, we confirmed that the privacy of the participants was maintained as the data were anonymized and confidentiality was maintained. Our study fully complied with the Declaration of Helsinki.

\section{Results}

During the period of the study, there were 3856 cesarean sections resulting in 3599 eligible women who underwent either elective or emergency cesarean section. Among all participants, there were only 338 primigravid women. There were no major complications or maternal mortality.

There were 2348 elective and 1251 emergency cesarean sections.

Among the elective cesarean section category, 85, 778 and 1485 were done under epidural, general and spinal anesthesia, respectively (see Table 1).

Maternal age was not statistically different among the three types of anesthesia in the elective category (see Table 2).

Previous history of cesarean section was not statistically different between the three types of anesthesia in both categories.

The mean age \pm standard deviation (SD) of women in the elective category was $33.1( \pm 5.5)$ years, and this was not statistically different among the anesthesia types. 
Table I Comparison of Different Anesthesia Types in Relation to Different Feto-Maternal Variables in the Elective Cesarean Section Category

\begin{tabular}{|c|c|c|c|c|c|}
\hline \multirow[t]{3}{*}{ Variables } & \multicolumn{5}{|c|}{ Elective Category } \\
\hline & \multirow{2}{*}{$\begin{array}{l}\text { Epidural } \\
\mathrm{N}=85\end{array}$} & \multirow{2}{*}{$\begin{array}{l}\text { General } \\
\mathbf{N}=778\end{array}$} & \multirow{2}{*}{$\begin{array}{l}\text { Spinal } \\
N=\mid 485\end{array}$} & \multirow{2}{*}{$\begin{array}{l}\text { Total } \\
\mathrm{N}=2348\end{array}$} & \multirow[t]{2}{*}{ P-value } \\
\hline & & & & & \\
\hline Age (years) mean (SD) & $33.4(5.2)$ & $33.2(5.2)$ & $33(5.7)$ & $33.1(5.5)$ & 0.4760 \\
\hline LOS (days) & $3.4(1.6)$ & $3.63(2.5)$ & $3.56(2.4)$ & $3.6(2.4)$ & 0.6140 \\
\hline Operative time (min) & $47.5(15.5)$ & $47.2(17.3)$ & $45.9(13.8)$ & $46.4(15.1)$ & 0.1230 \\
\hline Blood loss (mL) & $748.2(210)$ & $708.9(172.2)$ & $742.6(23 \mid .5)$ & $731(213.4)$ & 0.0010 \\
\hline Apgar-I minute & $8.0(0.0)$ & $7.9(0.58)$ & $7.9(0.480)$ & $7.9(0.5 \mathrm{I})$ & 0.0400 \\
\hline Apgar-5 minute & $9.0(0.0)$ & $8.99(0.13)$ & $8.97(0.419)$ & $8.9(0.34)$ & 0.5200 \\
\hline Diclofenac sodium & I.7I (I.I) & $1.73(1.3)$ & $2.05(1.5)$ & $1.93(1.5)$ & 0.0000 \\
\hline Morphine sulphate & $0.07(0.258)$ & $0.06(0.27)$ & $0.04(0.24)$ & $0.05(0.25)$ & 0.1420 \\
\hline Pethidine $\mathrm{HCL}$ & $1.49(0.88)$ & $1.79(1.2)$ & $1.55(1.1)$ & $1.63(1.1)$ & 0.0000 \\
\hline Paracetamol & $2.45(1.69)$ & $2.75(2.6)$ & $3.84(3.0)$ & $3.43(2.9)$ & 0.0000 \\
\hline Tramadol HCL & 0 & $0.024(0.29)$ & $0.01(0.17)$ & $0.02(0.22)$ & 0.3720 \\
\hline \multicolumn{6}{|l|}{ Previous CS } \\
\hline Yes & $20(23.5 \%)$ & $540(69.4 \%)$ & 915 (60.3\%) & 1475 (62.8\%) & $<0.0000$ \\
\hline No & 65 (76.5\%) & $238(30.6 \%)$ & 570 (39.7\%) & 873 (37.2\%) & \\
\hline \multicolumn{6}{|l|}{ NICU admission } \\
\hline Yes & 33 (38.8\%) & 387 (49.7\%) & 590 (39.7\%) & $1010(43.0 \%)$ & 0.0000 \\
\hline No & $52(61.2 \%)$ & $391(50.3 \%)$ & $895(60.3 \%)$ & I 338 (57.0\%) & \\
\hline
\end{tabular}

Abbreviations: SD, standard deviation; N, number; LOS, length of stay; min, minute; mL, milliliter; HCL, hydrochloride; CS, cesarean section; NICU, neonatal intensive care unit.

Regarding the elective cesarean category, compared with general anesthesia, there was a statistically significant difference reporting higher estimated blood loss (EBL) in spinal anesthesia, $P=0.001$. The mean EBL in this category was $731( \pm 213.4) \mathrm{mL}$. The mean APGAR score at 1 minute in the elective category was $7.9( \pm 0.51)$, with the highest score in epidural and lowest in general anesthesia. However, it was not statistically significant after performing Bonferroni test at $\mathrm{P}>0.05$. Furthermore, there was no statistically significant difference in the APGAR score at 5 minutes between the three different anesthesia types (see Tables 1 and 2).

In the emergency category, there were 314, 278 and 659 cesareans that were performed under epidural, general and spinal anesthesia, respectively (see Table 3 ).

The number of diclofenac sodium injections requested by the patients who had spinal anesthesia was higher than those who had general anesthesia, which was statistically significant $(\mathrm{P}=0.000)$. There was no statistically significant difference between epidural and general or between epidural and spinal anesthesia.

Regarding the number of pethidine HCL injections, these were requested more by the patients who had general than those who had spinal anesthesia, a statistically significant difference (the mean was $1.79( \pm 1.245), \mathrm{P}=$ 0.000). However, this was not statistically different between spinal and epidural anesthesia.

Paracetamol injections were requested mostly by patients who had spinal anesthesia (the mean number of injections was $3.84( \pm 3.0)$. This difference was statistically significant $(\mathrm{p}=0.000)$ compared with both epidural and general anesthesia. There was no statistically significant difference between epidural and general anesthesia $(\mathrm{P}=1.000)$.

The number of morphine sulfate injections was not statistically different among the three different types of anesthesia.

There was no statistically significant difference between the anesthesia types in relation to LOS, operative time or NICU admission (see Tables 1 and 2).

The mean age of women under the emergency anesthesia category was $32( \pm 5.7)$ years. Women in the spinal group were older than those belonging to the epidural group $(\mathrm{P}=0.000)$. Women were also statistically significantly older in the general than in the epidural group $(\mathrm{P}=0.016)$. 
Table 2 Multiple Comparisons Using Bonferroni Test; Elective Category

\begin{tabular}{|c|c|c|c|c|c|c|c|}
\hline \multirow[t]{2}{*}{ Dependent Variables } & \multirow[t]{2}{*}{ Anesthesia } & \multirow[t]{2}{*}{ Anesthesia } & \multirow[t]{2}{*}{ Mean Difference } & \multirow[t]{2}{*}{ Std. Error } & \multirow[t]{2}{*}{ Sig. } & \multicolumn{2}{|c|}{ 95\% Confidence Interval } \\
\hline & & & & & & Lower Bound & Upper Bound \\
\hline \multirow[t]{6}{*}{ LOS (days) } & Epidural & General & -.249 & 0.278 & 1.000 & -.92 & 0.42 \\
\hline & & Spinal & -.182 & 0.271 & 1.000 & -.83 & 0.47 \\
\hline & General & Epidural & 0.249 & 0.278 & 1.000 & -.42 & 0.92 \\
\hline & & Spinal & 0.068 & 0.108 & 1.000 & -.19 & 0.33 \\
\hline & Spinal & Epidural & 0.182 & 0.271 & 1.000 & -.47 & 0.83 \\
\hline & & General & -.068 & 0.108 & 1.000 & -.33 & 0.19 \\
\hline \multirow[t]{6}{*}{ Age (years) } & Epidural & General & 0.144 & 0.631 & 1.000 & -1.37 & 1.66 \\
\hline & & Spinal & 0.413 & 0.616 & 1.000 & -1.06 & 1.89 \\
\hline & General & Epidural & -.144 & 0.631 & 1.000 & -1.66 & 1.37 \\
\hline & & Spinal & 0.269 & 0.245 & 0.815 & -.32 & 0.86 \\
\hline & Spinal & Epidural & -.413 & 0.616 & 1.000 & -1.89 & 1.06 \\
\hline & & General & -.269 & 0.245 & 0.815 & -.86 & 0.32 \\
\hline \multirow{6}{*}{$\begin{array}{l}\text { Operative time } \\
\text { (minutes) }\end{array}$} & Epidural & General & 0.347 & 1.728 & 1.000 & -3.79 & 4.49 \\
\hline & & Spinal & 1.631 & 1.687 & 1.000 & -2.41 & 5.67 \\
\hline & General & Epidural & -.347 & 1.728 & 1.000 & -4.49 & 3.79 \\
\hline & & Spinal & 1.284 & 0.669 & 0.165 & -.32 & 2.89 \\
\hline & Spinal & Epidural & -1.631 & 1.687 & 1.000 & -5.67 & 2.41 \\
\hline & & General & -1.284 & 0.669 & 0.165 & -2.89 & 0.32 \\
\hline \multirow[t]{6}{*}{$\mathrm{EBL}(\mathrm{mL})$} & Epidural & General & 39.302 & 24.322 & 0.319 & -18.97 & 97.57 \\
\hline & & Spinal & 5.676 & 23.745 & 1.000 & -51.21 & 62.56 \\
\hline & General & Epidural & -39.302 & 24.322 & 0.319 & -97.57 & 18.97 \\
\hline & & Spinal & -33.626 & 9.423 & 0.001 & -56.20 & -11.05 \\
\hline & Spinal & Epidural & -5.676 & 23.745 & 1.000 & -62.56 & 51.21 \\
\hline & & General & 33.626 & 9.423 & 0.001 & 11.05 & 56.20 \\
\hline \multirow[t]{6}{*}{ APGAR score-Im } & Epidural & General & 0.100 & 0.058 & 0.252 & -.04 & 0.24 \\
\hline & & Spinal & 0.050 & 0.057 & 1.000 & -.09 & 0.19 \\
\hline & General & Epidural & -.100 & 0.058 & 0.252 & -.24 & 0.04 \\
\hline & & Spinal & -.050 & 0.022 & 0.075 & -.10 & 0.00 \\
\hline & Spinal & Epidural & -.050 & 0.057 & 1.000 & -.19 & 0.09 \\
\hline & & General & 0.050 & 0.022 & 0.075 & 0.00 & 0.10 \\
\hline \multirow[t]{6}{*}{ APGAR score- $5 \mathrm{~m}$} & Epidural & General & 0.010 & 0.039 & 1.000 & -.08 & 0.10 \\
\hline & & Spinal & 0.026 & 0.038 & 1.000 & -.07 & 0.12 \\
\hline & General & Epidural & -.010 & 0.039 & 1.000 & -.10 & 0.08 \\
\hline & & Spinal & 0.015 & 0.015 & 0.938 & -.02 & 0.05 \\
\hline & Spinal & Epidural & -.026 & 0.038 & 1.000 & -.12 & 0.07 \\
\hline & & General & -.015 & 0.015 & 0.938 & -.05 & 0.02 \\
\hline \multirow[t]{6}{*}{ Diclofenac sodium } & Epidural & General & -.024 & 0.165 & 1.000 & -.42 & 0.37 \\
\hline & & Spinal & -.341 & 0.161 & 0.104 & -.73 & 0.05 \\
\hline & General & Epidural & 0.024 & 0.165 & 1.000 & -.37 & 0.42 \\
\hline & & Spinal & -.316 & 0.064 & 0.000 & -.47 & -.16 \\
\hline & Spinal & Epidural & 0.341 & 0.161 & 0.104 & -.05 & 0.73 \\
\hline & & General & 0.316 & 0.064 & 0.000 & 0.16 & 0.47 \\
\hline
\end{tabular}

(Continued) 
Table 2 (Continued).

\begin{tabular}{|c|c|c|c|c|c|c|c|}
\hline \multirow[t]{2}{*}{ Dependent Variables } & \multirow[t]{2}{*}{ Anesthesia } & \multirow[t]{2}{*}{ Anesthesia } & \multirow[t]{2}{*}{ Mean Difference } & \multirow[t]{2}{*}{ Std. Error } & \multirow[t]{2}{*}{ Sig. } & \multicolumn{2}{|c|}{ 95\% Confidence Interval } \\
\hline & & & & & & Lower Bound & Upper Bound \\
\hline \multirow[t]{6}{*}{ Morphine sulfate } & Epidural & General & 0.010 & 0.029 & 1.000 & -.06 & 0.08 \\
\hline & & Spinal & 0.030 & 0.028 & 0.851 & -.04 & 0.10 \\
\hline & General & Epidural & -.010 & 0.029 & 1.000 & -.08 & 0.06 \\
\hline & & Spinal & 0.020 & 0.011 & 0.220 & -.01 & 0.05 \\
\hline & Spinal & Epidural & -.030 & 0.028 & 0.851 & -.10 & 0.04 \\
\hline & & General & -.020 & 0.011 & 0.220 & -.05 & 0.01 \\
\hline \multirow[t]{6}{*}{ Pethidine HCL } & Epidural & General & -.296 & 0.129 & 0.066 & -.61 & 0.01 \\
\hline & & Spinal & -.060 & 0.126 & 1.000 & -.36 & 0.24 \\
\hline & General & Epidural & 0.296 & 0.129 & 0.066 & -.01 & 0.61 \\
\hline & & Spinal & 0.236 & 0.050 & 0.000 & 0.12 & 0.36 \\
\hline & Spinal & Epidural & 0.060 & 0.126 & 1.000 & -.24 & 0.36 \\
\hline & & General & -.236 & 0.050 & 0.000 & -.36 & -.12 \\
\hline \multirow[t]{6}{*}{ Paracetamol } & Epidural & General & -.300 & 0.322 & 1.000 & -1.07 & 0.47 \\
\hline & & Spinal & -1.390 & 0.314 & 0.000 & -2.14 & -.64 \\
\hline & General & Epidural & 0.300 & 0.322 & 1.000 & -.47 & 1.07 \\
\hline & & Spinal & -1.090 & 0.125 & 0.000 & -1.39 & -.79 \\
\hline & Spinal & Epidural & 1.390 & 0.314 & 0.000 & 0.64 & 2.14 \\
\hline & & General & 1.090 & 0.125 & 0.000 & 0.79 & 1.39 \\
\hline \multirow[t]{6}{*}{ Tramadol HCL } & Epidural & General & -.0244 & 0.0247 & 0.966 & -.084 & 0.035 \\
\hline & & Spinal & -.0128 & 0.0241 & 1.000 & -.070 & 0.045 \\
\hline & General & Epidural & 0.0244 & 0.0247 & 0.966 & -.035 & 0.084 \\
\hline & & Spinal & 0.0116 & 0.0096 & 0.671 & -.011 & 0.035 \\
\hline & Spinal & Epidural & 0.0128 & $0.024 I$ & 1.000 & -.045 & 0.070 \\
\hline & & General & -.0116 & 0.0096 & 0.671 & -.035 & 0.011 \\
\hline
\end{tabular}

Abbreviations: SD, standard deviation; Std, standard; N, number; LOS, length of stay; mL, milliliter; HCL, hydrochloride; CS, cesarean section; NICU, neonatal intensive care unit; Sig, significant.

The mean of operation time was $44.4( \pm 16.4)$ minutes. The operation time using general anesthesia was longer than with epidural anesthesia and this was statistically significant $(P=0.042)$. However, no statistically significant difference was found between general and spinal or spinal and epidural.

Under the emergency category, the EBL was higher with general than with spinal or epidural anesthesia. This difference was statistically significant $(\mathrm{P}=0.000)$. There was no significant difference between spinal and epidural.

APGAR score at 1 and 5 minutes was higher with spinal than with general or epidural anesthesia, however, this difference was only statistically significant between spinal and general $(\mathrm{P}=0.000$ and 0.026 at 1 and 5 minutes, respectively), but there was no statistically significant difference between spinal and epidural anesthesia.
The mean number of diclofenac sodium injections in the emergency category was $2.13( \pm 1.8)$. After analysis with Bonferroni test, there was no statistically significant difference between the three different anesthetic modes.

Morphine sulfate injections were requested by more patients following general than either spinal or epidural anesthesia. This was statistically significant $(P=0.000$ in both). There was no statistically significant difference between spinal and epidural anesthesia.

There was no statistically significant difference in the NICU admission or in the LOS between the three different anesthesia types in the emergency category (see Tables 3 and 4).

We conducted phone interviews with the patients and asked them about their satisfaction with the type of anesthetic they had and whether they would recommend 
Table 3 Comparison of Different Anesthesia Types in Relation to Different Feto-Maternal Outcomes in the Emergency Cesarean Section Category

\begin{tabular}{|c|c|c|c|c|c|}
\hline \multirow[t]{3}{*}{ Variables } & \multicolumn{5}{|c|}{ Emergency Category } \\
\hline & \multirow{2}{*}{$\begin{array}{l}\text { Epidural } \\
\mathrm{N}=314\end{array}$} & \multirow{2}{*}{$\begin{array}{l}\text { General } \\
N=278\end{array}$} & \multirow{2}{*}{$\begin{array}{l}\text { Spinal } \\
N=659\end{array}$} & \multirow{2}{*}{$\begin{array}{l}\text { Total } \\
N=|25|\end{array}$} & \multirow[t]{2}{*}{ P-value } \\
\hline & & & & & \\
\hline Age; mean (SD) & $30.6(5.5)$ & $31.9(5.7)$ & $32.5(5.6)$ & $32(5.7)$ & 0.0000 \\
\hline LOS (days) & $3.6(2.6)$ & $3.2(2.1)$ & $3.5(2.4)$ & $3.5(2.4)$ & 0.1370 \\
\hline Operative time (min) & $43.03(13.9)$ & $46.4(21.0)$ & $44.2(15.2)$ & $44.4(16.4)$ & 0.0430 \\
\hline $\mathrm{EBL}(\mathrm{mL})$ & $730.3(184.7)$ & $877.3(709)$ & $733.1(213.5)$ & $759.2(385.0)$ & 0.0000 \\
\hline Apgar-I min & $7.7(I . I)$ & $7.5(1.4)$ & $7.88(0.67)$ & $7.76(0.97)$ & 0.0000 \\
\hline Apgar-5 min & $8.9(0.43)$ & $8.9(0.68)$ & $8.98(0.37)$ & $8.95(0.47)$ & 0.0280 \\
\hline Diclofenac sodium & $2.1(1.7)$ & $1.96(1.48)$ & $2.3(1.9)$ & $2.13(1.8)$ & 0.0420 \\
\hline Morphine sulphate & $0.02(0.25)$ & $0.15(0.47)$ & $0.03(0.19)$ & $0.05(0.294)$ & 0.0000 \\
\hline Pethidine HCL & $1.75(1.53)$ & $1.62(1.4)$ & $1.6(1.4)$ & $\mathrm{I} .64(\mathrm{I} .4)$ & 0.2810 \\
\hline Paracetamol & $4.3(3.2)$ & $4.3(4.5)$ & $4.5(3.5)$ & $4.4(3.7)$ & 0.6350 \\
\hline Tramadol HCL & $0.03(0.28)$ & $0.02(0.16)$ & $0.02(0.14)$ & $0.02(0.19)$ & 0.5110 \\
\hline \multicolumn{6}{|l|}{ Previous CS } \\
\hline Yes & 47 (15\%) & $108(38.8 \%)$ & 326 (49.5\%) & 481 (38.4\%) & 0.0000 \\
\hline No & 267 (85\%) & 170 (6I.2\%) & 333 (50.5\%) & $770(61.6 \%)$ & \\
\hline \multicolumn{6}{|l|}{ NICU admission } \\
\hline Yes & 143 (45.5\%) & $130(46.8 \%)$ & 277 (42.0\%) & 550 (44.0\%) & 0.3330 \\
\hline No & $17 \mid(54.5 \%)$ & I 48 (53.2\%) & 382 (58.0\%) & $701(56.0 \%)$ & \\
\hline
\end{tabular}

Abbreviations: SD, standard deviation; $\mathrm{N}$, number; LOS, length of stay; min, minute; EBL, estimated blood loss; $\mathrm{mL}$, milliliter; HCL, hydrochloride; CS, cesarean section; $\mathrm{NICU}$, neonatal intensive care unit.

it for a friend. Of the elective category, 781 patients could be reached. More patients were satisfied with the general anesthesia than the spinal or epidural and this was statistically significant $(\mathrm{P}=0.000)$. Also, more patients were satisfied with spinal than with epidural and they would recommend spinal rather than epidural anesthesia. This result was also statistically significant $(\mathrm{P}=0.000)($ see Table 5).

In terms of the emergency category, we obtained the views of 284 women. A significantly higher percentage of patients were satisfied with and would recommend epidural $(\mathrm{P}=0.000)$ or spinal $(\mathrm{P}=0.004)$ rather than general anesthesia. A statistically significant percentage of patients were satisfied with, would recommend, epidural rather than spinal anesthesia $(\mathrm{P}=0.004)$ (see Table 5).

\section{Discussion}

We made the study population as homogeneous as possible by dividing the cesarean sections into two categories, elective and emergency. We compared the different anesthesia types within each category separately. Furthermore, the results show that the women under each category were not significantly different from maternal age and previous cesarean section(s) perspectives. These features allowed a reasonable comparison and gave credible results.

With the exception of the EBL and some analgesia requirements, there were no significant differences between all three anesthesia types in the elective cesarean sections.

This study did not rely on sophisticated method(s) for EBL, but the method of estimation was unified in all cesareans. In women with high EBL, Rubenstein et al. ${ }^{10}$ found that using a novel colorimetric device was a more accurate predictor of post-operative hemoglobin. Other authors ${ }^{11}$ did not support the view that EBL or quantitative assessment of blood loss (QBL) methods accurately reflect the actual blood loss during scheduled cesarean section. The EBL in our study was highest in the SA, and this was higher than in the GA. Alternatively, Aksoy et al. ${ }^{12}$ in a prospective study found that GA was associated with a higher risk of operative blood loss than SA in low-risk patients undergoing elective CS. These authors used preoperative, post-operative hemoglobin, and hematocrit for comparison. The difference in EBL in our study was very minimal and might not be reflected in a significant 
Table 4 Multiple Comparisons Using Bonferroni Test; Emergency Category

\begin{tabular}{|c|c|c|c|c|c|c|c|}
\hline \multirow[t]{2}{*}{ Dependent Variables } & \multirow[t]{2}{*}{ Anesthesia } & \multirow[t]{2}{*}{ Anesthesia } & \multirow[t]{2}{*}{ Mean Difference } & \multirow[t]{2}{*}{ Std. Error } & \multirow[t]{2}{*}{ Sig. } & \multicolumn{2}{|c|}{ 95\% Confidence Interval } \\
\hline & & & & & & Lower Bound & Upper Bound \\
\hline \multirow[t]{6}{*}{ LOS (days) } & Epidural & General & 0.378 & 0.195 & 0.160 & -.09 & 0.85 \\
\hline & & Spinal & 0.111 & 0.163 & 1.000 & -.28 & 0.50 \\
\hline & General & Epidural & -.378 & 0.195 & 0.160 & -.85 & 0.09 \\
\hline & & Spinal & -.267 & 0.170 & 0.347 & -.67 & 0.14 \\
\hline & Spinal & Epidural & -.111 & 0.163 & 1.000 & -.50 & 0.28 \\
\hline & & General & 0.267 & 0.170 & 0.347 & -.14 & 0.67 \\
\hline \multirow[t]{6}{*}{ Age (years) } & Epidural & General & -1.290 & 0.464 & 0.016 & -2.40 & -.18 \\
\hline & & Spinal & -1.926 & 0.386 & 0.000 & -2.85 & -1.00 \\
\hline & General & Epidural & 1.290 & 0.464 & 0.016 & 0.18 & 2.40 \\
\hline & & Spinal & -.636 & 0.403 & 0.342 & -1.60 & 0.33 \\
\hline & Spinal & Epidural & 1.926 & 0.386 & 0.000 & 1.00 & 2.85 \\
\hline & & General & 0.636 & 0.403 & 0.342 & -.33 & 1.60 \\
\hline \multirow{6}{*}{$\begin{array}{l}\text { Operative time } \\
\text { (minutes) }\end{array}$} & Epidural & General & -3.328 & 1.350 & 0.042 & -6.56 & -.09 \\
\hline & & Spinal & -1.131 & 1.124 & 0.945 & -3.83 & 1.56 \\
\hline & General & Epidural & 3.328 & 1.350 & 0.042 & 0.09 & 6.56 \\
\hline & & Spinal & 2.197 & 1.173 & 0.184 & -.61 & 5.01 \\
\hline & Spinal & Epidural & 1.131 & 1.124 & 0.945 & -1.56 & 3.83 \\
\hline & & General & -2.197 & 1.173 & 0.184 & -5.01 & 0.61 \\
\hline \multirow[t]{6}{*}{$\mathrm{EBL}(\mathrm{mL})$} & Epidural & General & -147.083 & 31.300 & 0.000 & -222.12 & -72.05 \\
\hline & & Spinal & 7.190 & 26.063 & 1.000 & -55.29 & 69.67 \\
\hline & General & Epidural & | 47.083 & 31.300 & 0.000 & 72.05 & 222.12 \\
\hline & & Spinal & 154.273 & 27.182 & 0.000 & 89.11 & 219.43 \\
\hline & Spinal & Epidural & -7.190 & 26.063 & 1.000 & -69.67 & 55.29 \\
\hline & & General & -154.273 & 27.182 & 0.000 & -219.43 & -89.11 \\
\hline \multirow[t]{6}{*}{ APGAR score-Im } & Epidural & General & 0.180 & 0.079 & 0.071 & -.01 & 0.37 \\
\hline & & Spinal & -.155 & 0.066 & 0.056 & -.31 & 0.00 \\
\hline & General & Epidural & -.180 & 0.079 & 0.071 & -.37 & 0.01 \\
\hline & & Spinal & -.335 & 0.069 & 0.000 & -.50 & -.17 \\
\hline & Spinal & Epidural & 0.155 & 0.066 & 0.056 & 0.00 & 0.31 \\
\hline & & General & 0.335 & 0.069 & 0.000 & 0.17 & 0.50 \\
\hline \multirow[t]{6}{*}{ APGAR score-5m } & Epidural & General & 0.048 & 0.039 & 0.654 & -.05 & 0.14 \\
\hline & & Spinal & -.041 & 0.032 & 0.616 & -.12 & 0.04 \\
\hline & General & Epidural & -.048 & 0.039 & 0.654 & -.14 & 0.05 \\
\hline & & Spinal & -.089 & 0.034 & 0.026 & -.17 & -.01 \\
\hline & Spinal & Epidural & 0.041 & 0.032 & 0.616 & -.04 & 0.12 \\
\hline & & General & 0.089 & 0.034 & 0.026 & 0.01 & 0.17 \\
\hline \multirow[t]{6}{*}{ Diclofenac sodium } & Epidural & General & 0.088 & 0.147 & 1.000 & -.26 & 0.44 \\
\hline & & Spinal & -.206 & 0.122 & 0.276 & -.50 & 0.09 \\
\hline & General & Epidural & -.088 & 0.147 & 1.000 & -.44 & 0.26 \\
\hline & & Spinal & -.294 & 0.127 & 0.064 & -.60 & 0.01 \\
\hline & Spinal & Epidural & 0.206 & 0.122 & 0.276 & -.09 & 0.50 \\
\hline & & General & 0.294 & 0.127 & 0.064 & -.01 & 0.60 \\
\hline
\end{tabular}

(Continued) 
Table 4 (Continued).

\begin{tabular}{|c|c|c|c|c|c|c|c|}
\hline \multirow[t]{2}{*}{ Dependent Variables } & \multirow[t]{2}{*}{ Anesthesia } & \multirow[t]{2}{*}{ Anesthesia } & \multirow[t]{2}{*}{ Mean Difference } & \multirow[t]{2}{*}{ Std. Error } & \multirow[t]{2}{*}{ Sig. } & \multicolumn{2}{|c|}{ 95\% Confidence Interval } \\
\hline & & & & & & Lower Bound & Upper Bound \\
\hline \multirow[t]{6}{*}{ Morphine sulfate } & Epidural & General & -.132 & 0.024 & 0.000 & -.19 & -.08 \\
\hline & & Spinal & -.005 & 0.020 & 1.000 & -.05 & 0.04 \\
\hline & General & Epidural & 0.132 & 0.024 & 0.000 & 0.08 & 0.19 \\
\hline & & Spinal & 0.127 & 0.021 & 0.000 & 0.08 & 0.18 \\
\hline & Spinal & Epidural & 0.005 & 0.020 & 1.000 & -.04 & 0.05 \\
\hline & & General & -.127 & 0.021 & 0.000 & -.18 & -.08 \\
\hline \multirow[t]{6}{*}{ Pethidine HCL } & Epidural & General & 0.133 & 0.116 & 0.755 & -.14 & 0.41 \\
\hline & & Spinal & 0.151 & 0.097 & 0.356 & -.08 & 0.38 \\
\hline & General & Epidural & -.133 & 0.116 & 0.755 & -.41 & 0.14 \\
\hline & & Spinal & 0.018 & 0.101 & 1.000 & -.22 & 0.26 \\
\hline & Spinal & Epidural & -.151 & 0.097 & 0.356 & -.38 & 0.08 \\
\hline & & General & -.018 & 0.101 & 1.000 & -.26 & 0.22 \\
\hline \multirow[t]{6}{*}{ Paracetamol } & Epidural & General & -.023 & 0.305 & 1.000 & -.75 & 0.71 \\
\hline & & Spinal & -.210 & 0.254 & 1.000 & -.82 & 0.40 \\
\hline & General & Epidural & 0.023 & 0.305 & 1.000 & -.71 & 0.75 \\
\hline & & Spinal & -.187 & 0.264 & 1.000 & -.82 & 0.45 \\
\hline & Spinal & Epidural & 0.210 & 0.254 & 1.000 & -.40 & 0.82 \\
\hline & & General & 0.187 & 0.264 & 1.000 & -.45 & 0.82 \\
\hline \multirow[t]{6}{*}{ Tramadol HCL } & Epidural & General & 0.0107 & 0.0156 & 1.000 & -.027 & 0.048 \\
\hline & & Spinal & 0.0127 & 0.0130 & 0.981 & -.018 & 0.044 \\
\hline & General & Epidural & -.0107 & 0.0156 & 1.000 & -.048 & 0.027 \\
\hline & & Spinal & 0.0021 & 0.0135 & 1.000 & -.030 & 0.035 \\
\hline & Spinal & Epidural & -.0127 & 0.0130 & 0.981 & -.044 & 0.018 \\
\hline & & General & -.0021 & 0.0135 & 1.000 & -.035 & 0.030 \\
\hline
\end{tabular}

Abbreviations: SD, standard deviation; Std, standard; N, number; LOS, length of stay; $\mathrm{mL}$, milliliter; HCL, hydrochloride; CS, cesarean section; NICU, neonatal intensive care unit; Std, standard; Sig, significant.

difference in hemoglobin; the means were 708.9 ( \pm $172.2)$ and $742.6( \pm 231.5)$ for GA and SA, respectively. The difference in the mean was only $34 \mathrm{~mL}$.
Regarding post-operative analgesia, SA patients requested more diclofenac sodium and less pethidine HCL than those who had GA.

Table 5 Patients' Satisfaction with Anesthetic Type

\begin{tabular}{|c|c|c|c|c|c|c|}
\hline \multirow[t]{2}{*}{ Satisfaction } & \multicolumn{3}{|l|}{ Elective } & \multicolumn{3}{|c|}{ Emergency } \\
\hline & Yes & No & $P$ value & Yes & No & $P$ value \\
\hline Epidural; N (\%) & $\mathrm{I}(2.70)$ & $37(97.4)$ & 0.000 & $45((37.5)$ & $75(62.5)$ & 0.000 \\
\hline General; N (\%) & $83(30.6)$ & $188(69.4)$ & & $10(11.2)$ & 79(88.8) & \\
\hline Epidural; N (\%) & $\mathrm{I}(2.7)$ & $37(97.4)$ & 0.001 & $45(37.5)$ & $75(62.5)$ & 0.004 \\
\hline Spinal; N (\%) & $61(12.9)$ & $4 \mid I(87 . I)$ & & $46(26.3)$ & $129(73.7)$ & \\
\hline General; N (\%) & $83(30.6)$ & $188(69.4)$ & 0.000 & $10(11.2)$ & $79(88.8)$ & 0.004 \\
\hline Spinal; N (\%) & $6 \mathrm{I}(12.9)$ & $4 I I(87.1)$ & & $46(26.3)$ & I $29(73.7$ & \\
\hline
\end{tabular}

Abbreviations: N, number; \%, percentage. 
With the exception of paracetamol, hich was used more frequently by SA patients, there was no significant difference between SA and EA in the elective CS regarding post-operative analgesic requirements. Our finding of higher use of paracetamol and diclofenac sodium in the SA patients could be explained by the fact that a group of these patients suffered post-operative headache, and because they did not want to be sedated in order to keep their newborns with them. A similar feature of SA was also concluded by Schewe et al. ${ }^{13}$ They found that SA was associated with less pain post-operatively, less use of additional analgesics and fewer side-effects. They also found that patient-controlled analgesia (PCA) was similar after SA and EA.

In our hospital, unfortunately, we did not offer PCA. Moreover, there was no well-defined post-operative analgesia protocol. There two factors could have contributed to bias in analgesic requirements by the different patients.

The GA patients in our study requested more pethidine than SA patients. Saracoglu et al. ${ }^{14}$ found that the number of patients requiring opioid via PCA in the first 24 hours was significantly higher in the GA group. Also, the patient satisfaction was significantly higher in the SA group. We found a similar result in the emergency category; morphine sulfate was used by more GA patients than SA or EA. This difference was statistically significant.

The SA in our study involved the use of fentanyl. El Aish et al. ${ }^{15}$ found that although morphine was associated with more side effects than fentanyl, spinal morphine was more effective than spinal fentanyl for cesarean analgesia.

APGAR scores at both 1 and 5 minutes were not significantly different between the three different types of anesthesia in the elective cesarean sections while in the emergency cesarean section category, APGAR scores at 1 and 5 minutes were higher in the SA patients than GA, and this was statistically significant. This could be due to intra-uterine fetal distress not related to the GA, to the opioids used in the GA or to a combination of these factors. This difference was not reflected in a statistically significant difference in NICU admission. There was no significant difference between SA and EA. These fetal outcomes were similar to a network meta-analysis of 46 randomized trials involving 3689 patients that showed significant differences in Apgar score $\leq 6$ at 1 minute between SA versus GA and Apgar scores at 1 - and 5-min, in favor of SA. ${ }^{16}$ Dyer et al. ${ }^{17}$ affirmed that 1-min Apgar scores were lower after GA than after SA in patients who had preeclampsia and non- reassuring fetal heart traces. They also found no difference in the number of patients with Apgar scores less than 7 at 1 or 5 minutes or umbilical arterial $\mathrm{pH}$ less than 7.2 or in the requirements for resuscitation. In our study, we did not find a statistically significant difference in the NICU admission across the three different anesthesia types in both elective and emergency categories. Whether the cesarean section was elective or emergency, our study indicated a similar clinical neonatal safety profile among SA, GA and EA.

The LOS in the hospital and operative time were not significantly different between the three types of anesthesia in the elective cesarean sections. In the emergency category, the LOS was not statistically different between any anesthetic but the operative time was longer in the GA than the EA with no significant difference between GA and SA or SA and EA. The long mean operative time in the GA in the emergency category was due to prolonged muscle relaxant effect and delayed extubation.

In a prospective observational study of 61 patients undergoing emergency cesarean section for fetal distress under either GA or regional anesthesia, Edipoglu et al. ${ }_{-}^{18}$ found no statistical evidence that either anesthesia technique is superior regarding neonatal morbidity; the 1-minute Apgar score was significantly lower with GA, but this was not the case for the 5-minute Apgar score and regional anesthesia was non-significantly associated with shorter length of hospitalization.

In a quasi-controlled trial in our region, Gaza, Tafish et al. ${ }^{19}$ reported that there was no difference between GA and SA in LOS, operative time and time to demand for analgesia. They concluded that GA and SA had a similar safety profile. Ikeda et al., ${ }^{20}$ in a retrospective review, found a trend towards fewer obstetric GA since 2015. In our study, $29.3 \%$ of all cesarean sections (both elective and emergency) were done under GA.

In our study, the patients who had elective cesarean sections were satisfied with and would recommend GA rather than regional anesthesia. However, those who had emergency cesarean sections were satisfied with and would recommend regional anesthesia. They indicated that, in the elective group, the fear and accompanied anxiety were the main reasons behind that choice. Our hospital policy did not allow the partner or a relative to be with and support the women in theatre so they preferred to be asleep. Moreover, our neonatal policy did not allow immediate handling of the newborns by their mothers in theatre. Some women also indicated that they did not receive enough post-cesarean analgesia after SA or EA. 
In the emergency category, the women were anxious about their babies, and they would prefer to be awake at the time of delivery. To maximize the post-operative analgesic needs of women, different types of analgesics were used including non-steroidal anti-inflammatory drugs. Good and adequate pain management should be an important priority for women having cesarean section. That is because suboptimal post-operative pain management was proved to be associated with chronic pain, higher opioid use, slow recovery to normal functions, impaired maternal-fetal bonding, and increased risk of postpartum depression. Use of multimodal analgesics with involvement of patients' views, should be the core principle for cesarean section post-operative pain management. ${ }^{21}$ It is recommended to have a component of relief that childbirth was over and safe and there might be distress on seeing the sex of child in our society; these, irrespective of the mode of anesthesia, would have contributed to the overall satisfaction.

Chen et al. ${ }^{22}$ found that, compared with SA, the GA group was significantly higher in post-operative patient satisfaction. However, Ghaffari et al. ${ }^{23}$ found that SA, compared with GA, was the technique of choice for cesarean section because, in addition to other benefits, it provided effective pain control, mobility and fast return back to daily activities for new mothers and increased their quality of life. Around $87 \%$ of our patients who had elective cesarean section were not satisfied with and did not recommend SA due to post-operative pain and they indicated that the post-operative analgesia was not offered adequately. In a prospective study of women who underwent cesarean section under SA, $68 \%$ of participants reported they were satisfied with their pain control. ${ }^{24}$ High-quality health care and anesthesia with pain management must be assessed to determine patient satisfaction. ${ }^{25}$ The patients' satisfaction in our study was a secondary outcome. Our main aim was to generally explore the patients' overall general views. This outcome could be studied formally and in detail and could be a whole subject on its own.

Our study had a relatively large number of patients. The three types of anesthesia were compared in both elective and emergency cesarean sections. We also studied a significant and important number of feto-maternal outcomes.

Our study was limited by its retrospective nature and the potential confounding factors such as the experience of the anesthetists/obstetricians and associated maternal morbidity factors. Moreover, as EA was recommended mainly for patients in labor or undergoing induction of labor, and we did not keep the epidural catheters post-operatively, there was a small number of elective cesarean sections performed under EA. We recommend extensive further studies taking into consideration patients' satisfaction with the mode of anesthesia using standardized objective assessment methods to eliminate confounding factors contributing to different bias.

\section{Conclusions}

There was no statistically significant difference among the three types of anesthesia regarding neonatal intensive care admission and length of hospital stay for emergency and elective categories. APGAR score was higher with spinal than with general anesthesia in the emergency category with no significant difference in the elective category. More diclofenac sodium and paracetamol and less opioids were used after regional than after general anesthesia. Satisfaction was higher with epidural anesthesia.

\section{Abbreviations}

$\mathrm{SD}$, standard deviation; N, number; LOS, length of stay; min, minute; $\mathrm{mL}$; milliliter; HCL, hydrochloride; CS, cesarean section; NICU, neonatal intensive care unit.

\section{Consent}

Written Informed Consents; not applicable as per IRB due to retrospective nature.

\section{Disclosure}

All authors declare no conflicts of interests for this work.

\section{References}

1. James AH, Jamison MG, Brancazio LR, Myers ER. Venous thromboembolism during pregnancy and the postpartum period: incidence, risk factors, and mortality. Am J Obstetric Gynecol. 2006;194 (5):1311-1315. doi:10.1016/j.ajog.2005.11.008

2. James AH. Pregnancy and thrombotic risk. Crit Care Med. 2010;38:57-63. doi:10.1097/CCM.0b013e3181c9e2bb

3. Munnur U, de Boisblanc B, Suresh MS. Airway problems in pregnancy. Crit Care Med. 2005;33:259-268. doi:10.1097/01. ccm.0000183502.45419.c9

4. Kinsella SM, Lohmann G. Supine hypotensive syndrome. Obstetric Gynecol. 1994;83:774-788.

5. Kinsella SM, Walton B, Sashidharan R, et al. Category-1 caesarean section: a survey of anaesthetic and peri-operative management in the UK. Anaesthesia. 2010;65:362-368. doi:10.1111/j.1365-2044.2010.06265.x

6. Devroe S, Van de Velde M, Rex S. General anesthesia for caesarean section. Curr Opin Anaesthesiol. 2015;28(3):240-246. doi:10.1097/ ACO.0000000000000185

7. Riley ET, Cohen SE, Macario A, Desai JB, Ratner EF. Spinal versus epidural anesthesia for cesarean section: a comparison of time efficiency, costs, charges, and complications. Anesth Analg. 1995;80 (4):709-712. doi:10.1097/00000539-199504000-00010 
8. Fettes PD, Jansson JR, Wildsmith JA. Failed spinal anaesthesia: mechanisms, management, and prevention. Br J Anaesth. 2009;102 (6):739-748. doi:10.1093/bja/aep096

9. Clark SL, Belfort MA, Dildy GA, Herbst MA, Meyers JA, Hankins GD. Maternal death in the 21st century: causes, prevention, and relationship to cesarean delivery. Am J Obstetric Gynecol. 2008;199(1):36 e1-5; discussion 91-2, e7-11. doi:10.1016/j. ajog.2008.03.007

10. Rubenstein AMD, Tully GMD, Thurer RMD. Accurate assessment of blood loss during cesarean delivery improves estimate of postoperative hemoglobin [17B]. Obstet Gynecol. 2018;131:p 23S-24S. doi:10.1097/01.AOG.0000532919.12355.64

11. Conly BMD, Sylla RMD, Lee KMD, Wei JMPH. Improved estimation of blood loss at time of cesarean section using a quantitative approach [28M]. Obstet Gynecol. 2017;129(5 - p S140). doi:10.1097/ 01.AOG.0000514699.18738.76

12. Aksoy H, Ü A, Yücel B, et al. Blood loss in elective cesarean section: is there a difference related to the type of anesthesia? A randomized prospective study. J Turk Ger Gynecol Assoc. 2015;16(3):158-163. doi:10.5152/jtgga.2015.15034

13. Schewe JC, Komusin A, Zinserling J, Nadstawek J, Hoeft A, Hering R. Effects of spinal anaesthesia versus epidural anaesthesia for caesarean section on postoperative analgesic consumption and postoperative pain. Eur J Anaesthesiol. 2009;26(1):52-59. doi:10.1097/EJA.0b013e328318c639

14. Saracoglu KT, Saracoglu A, Umuroglu T, Eti Z. Neuraxial block versus general anaesthesia for cesarean section: post-operative pain scores and analgesic requirements. J Pak Med Assoc. 2012;62(5):441-444.

15. El Aish KA, Tafish R, Zourob H. Morphine versus fentanyl for spinal post-caesarean analgesia: a randomised controlled trial. Lancet. 2018;21(391 Suppl 2):S20. doi:10.1016/S0140-6736(18)30386-6

16. Kim WH, Hur M, Park SK, et al. Comparison between general, spinal, epidural, and combined spinal-epidural anesthesia for cesarean delivery: a network meta-analysis. Int $J$ Obstet Anesth. 2019;37:5-15. doi:10.1016/j.ijoa.2018.09.012
17. Dyer RA, Els I, Farbas J, Torr GJ, Schoeman LK, James MF. Prospective, randomized trial comparing general with spinal anesthesia for cesarean delivery in preeclamptic patients with a nonreassuring fetal heart trace. Anesthesiology. 2003;99 (3):561-569. doi:10.1097/00000542-200309000-00010

18. Edipoglu IS, Celik F, Marangoz EC, Orcan GH. Effect of anaesthetic technique on neonatal morbidity in emergency caesarean section for foetal distress. PLoS One. 2018;13(11):e0207388. doi:10.1371/journal.pone. 0207388

19. Tafish R, KIA EA, Madi W. General versus spinal anaesthesia for caesarean section: a quasi-controlled trial. Lancet. 2018;21(391 Suppl 2):S33. doi:10.1016/S0140-6736(18)30399-4

20. Ikeda T, Kato A, Bougaki M, et al. A retrospective review of 10-year trends in general anesthesia for cesarean delivery at a university hospital: the impact of a newly launched team on obstetric anesthesia practice. $B M C$ Health Serv Res. 2020;20:421. doi:10.1186/s12913-020-05314-2

21. Carvalho B, Butwick AJ. Postcesarean delivery analgesia. Best Pract Res Clin Anaesthesiol. 2017;31(1):69-79. doi:10.1016/j.bpa.2017.01.003

22. Chen Y, Liu W, Gong X, Cheng Q. Comparison of effects of general anesthesia and combined spinal/epidural anesthesia for cesarean delivery on umbilical cord blood gas values: a double-blind, randomized, controlled study. Med Sci Monit. 2019;16(25):5272-5279. doi:10.12659/MSM.914160

23. Ghaffari S, Dehghanpisheh L, Tavakkoli F, Mahmoudi H. The effect of spinal versus general anesthesia on quality of life in women undergoing cesarean delivery on maternal request. Cureus. 2018;11 (10):12:e3715. doi:10.7759/cureus.3715

24. Kintu A, Abdulla S, Lubikire A, et al. Postoperative pain after cesarean section: assessment and management in a tertiary hospital in a low-income country. BMC Health Serv Res. 2019;19(1):68. doi:10.1186/s12913-019-3911-x

25. Burch T, Seipel SJ, Coyle N, Ortega KH, DeJesus O. Postoperative visual analog pain scores and overall anesthesia patient satisfaction. Crit Care Nurs Clin North Am. 2017;29(4):419-426. doi:10.1016/j.cnc.2017.08.003

\section{Publish your work in this journal}

The International Journal of Women's Health is an international, peerreviewed open-access journal publishing original research, reports, editorials, reviews and commentaries on all aspects of women's healthcare including gynecology, obstetrics, and breast cancer. The manuscript management system is completely online and includes a very quick and fair peer-review system, which is all easy to use. Visit http://www.dovepress.com/testimonials.php to read real quotes from published authors. 